\title{
Trauma and the Zero Process: Theoretical Considerations
}

\author{
Joseph Fernando \\ Toronto Institute of Psychoanalysis, Toronto, Canada
}

The basic idea, developed in a previous paper, of post traumatic zero process memories as something that behave more like lived present or future experiences is further elaborated. The nature of the perceptual apparatus of the mind, and the way zero process memories come to partially invade the perceptual and action apparatuses rather than living in the memory systems, is described. This invasion means that the traumatized person comes to live in two worlds, with the zero process memories playing out in the present and influencing their thoughts, feelings, and actions. The characteristics of the zero process are compared to those of the primary process, which Freud discovered as the very different way in which the deeper emotional mind works. While they are often confused, it is argued that the nature of the timelessness, reality distortion, and basic drives that can be seen in these two forms of functioning are quite different. Borderline personality disorder is briefly described as dominated by characteristics of the primary process, and is contrasted with post traumatic states, dominated by the zero process. With a deeper and more accurate understanding of the zero process, it can be seen that many things can have a zero process form, and a number of these are described: zero process defenses, zero process objects (introjects), zero process mourning, and zero process symptoms. The use of the concept of the zero process to comprehend intergenerational transmission of trauma is described. Psychoanalysis 2018;29(3):46-53

KEY WORDS: Trauma $\cdot$ Zero process $\cdot$ Primary process $\cdot$ Zero process defence.

Received: March 26, 2018 Accepted: May 1, 2018

Address for correspondence: Joseph Fernando, Mpsy, MD

Toronto Institute of Psychoanalysis, 40 St. Clair Ave. East, Toronto, ON M4T 1M9, Canada

Tel: +1-416-975-9147, E-mail: jfernsympatico@gmail.com

In the first paper of this series I presented basic conceptualizations about trauma and the zero process, illustrated by clinical material from two patients. I described the traumatic process as defined by the shutting down of many higher level mental functions. Because of this shutdown, the person is left with unprocessed memories that behave in many ways more like a present experience than a memory. In this lecture, I would like to describe further characteristics of the zero process, and their applications.

By all clinical indications, the core of the zero process seems to function as an experience. Freud (1925), in conceptualizing the perceptual apparatus, compared it to a slate that is wiped clean each time, ready for new impressions. Memory, by contrast, involves the creation of a more permanent formation. We now know that there are very transient immediate memories, somewhat longer lasting short term memories, and then the consolidation of some of these into various forms of long term memory. The basic idea, however, would seem to still hold. The perceptual apparatus of the mind has to be largely free of fixed formations such as memory, in or-

This is an Open Access article distributed under the terms of the Creative Commons Attribution Non-Commercial License (https://creativecommons.org/licenses/by-nc/4.0) which permits unrestricted non-commercial use, distribution, and reproduction in any medium, provided the original work is properly cited. der to register new impressions. However, in order to process these new impressions, the perceptual apparatus actually needs certain forms of memory. As an example, if the eyes do not provide input, for instance because of congenital cataracts, the visual cortex will not organize itself in infancy in order to be able to organize incoming perceptions and analyze them, for instance in terms of lines, shapes, etc. These seem like immediate input, but this kind of situation makes it clear that even at this most basic level, the perceptual apparatus of the mind does not merely receive these as givens, but has to create them. Perhaps more accurately we might say it analyzes the input and discovers reality, although what happens is something involving both creation and discovery. As the input from a sensory receptor moves into the mind, it is analyzed first for basic qualities, and then for more holistic, gestalt aspects. These later are more obviously related to past experience, for instance a specific face or a voice, but they are only the continuation of a process of analysis of the input and construction of the experience. By the time we have what we think of as our immediate experience of the reality, a good deal of this processing has already taken place. Our conscious experience takes place as we combine the outcome of this processing with memories and models of the world that we already possess. Slights of hand and illusions rely on the fact 
that our expectations create our experience at this level. And of course from this point on, processing of the experience still continues.

What we know is that trauma disrupts this processing of incoming sensory data, as well as the processing of other aspects of experience such as actions and feelings, at various points. At the less disruptive level, an upsetting or unpleasant experience may be resisted in the sense of trying not to recognize it and making it real-the sort of experience where the person will say, "this can't be happening". At a higher level of disruption you get the breakdown of the basic processing of the event, as described already in the clinical material of the last paper, leading to changes in level of consciousness, to lack of integration, etc. Some combination of suddenness of the event and of breakdown of filtering mechanisms leading to annihilation anxiety, causes a shutdown of the processing of incoming stimuli, along with the shutdown of other ego functions related to action and affect. Why external overwhelming leads to this kind of shutdown is not a question I think we have a final answer for. The answer may have to be sought more at the evolutionary than at the functional psychological level. It is clear that the basic traumatic response, including the formation of memories that behave more like experiences, is present at least among mammals, and perhaps in somewhat different form in some other animals. There are various theories related to freezing responses, over-activation, and the adaptiveness of creating indelible memories of dangerous situations. Powerful learning after only a single event is useful in extreme circumstances, and it may be that in trauma this mechanism, which we see in stressful and frightening events, overshoots the mark, leading to a kind of single event learning which is not so adaptive. Given that overwhelming events lead to a shutdown in the processing of an experience, such as Joyce's rape, or Cordelia being told about her mother's death, what we observe is that with a breakdown in the first order construction of the present experience, the memory of the event does not behave like a regular memory, but like an experience. We could ask why the truncated processing of the experience should lead to this outcome? Here too, I don't think we have a very good explanation of why this should be so. But we know that it is so. My theory is that the partially formed experience is not laid down in the usual long term memory systems of the mind, but in the perceptual and action areas instead. In other words, memory, in the sense of long term storage, invades those areas of the mind that usually subserve immediate perception and action.

The key aspect of the zero process to understand is that the realities related to trauma are present at all times, as a second reality that the person has to respond to. Because we become more directly aware of the zero process memories when re- living is triggered, as happened in the example with Joyce, it may be tempting to see them as more regular but very powerful memories that are evoked by experiences. Many observations argue against this view. When one analyzes more deeply, one finds that the person is in fact always reacting in subtle ways to this reality. Or, to phrase it more accurately, some of their actions, feelings, and beliefs are from that part of them that still lives in the other, traumatic, reality. Along with the present reality, Cordelia lived in a world where a woman was losing weight, had pains in her belly, had no appetite, and had a deadly disease that the doctors could not cure. This reality expressed itself in physical symptoms, feelings, and behaviour towards others. This other reality can make a person look psychotic at times, but it differs from true psychosis. We can distinguish zero process functioning from schizoid and psychotic disorders where there is a thin layer of normal functioning covering over a deeper break with reality, based on global, rather than focal, deficits in important ego functions. Niederland's freezing patient was misdiagnosed as psychotic because of the bizarreness of his symptom, but Niederland saw the more regular functioning and contact with reality, which proved not to be a facade but a true part of him, alongside which the patient also lived in the cold and frozen reality of his early trauma.

It is not only perceptions but also many affects, thoughts, and body reactions related to the trauma that are also part of the unprocessed past that lives in the present. This is especially shown by the importance of performing specific physical actions in the therapy of, and recovery from, trauma. The intention to act, when it becomes part of the zero process, has not only its characteristics of remaining a perpetual present, but also of remaining unintegrated with other actions and perceptions and judgement. The work of therapy is to bring this integration about. We should not forget that the intention to act, and actions themselves, are one of the key components of our mental functioning, and thus of the zero process traumatic moments that become a perpetual present.

The zero process has often been confused with the deeper emotional aspects of the mind that Freud called the primary process. Freud (1900) first introduced the idea of the primary process and described its characteristics in the Interpretation of Dreams, where he demonstrated how, through the method of free association, he could not only reveal hidden wishes and forgotten memories, but also a form of mental functioning that was different from that of which we are usually aware. By comparing the manifest dream with the repressed, latent dream thoughts, memories, and fantasies uncovered through the method of free association, Freud showed that the original thoughts had been subject to a number of changes. Some were related to factors such as disguise imposed by 
the censorship because the thoughts were unacceptable to the person, but a number of the processes, which occurred in all cases, and also in the formation of neurotic symptoms, jokes, and artistic productions, to name a few, led Freud to infer that the thoughts had been subjected in the unconscious to very different mental processes to those of which we are usually aware. He called this the primary process, implying that it was there from the start, only to be later overlaid by the more reality oriented and organized secondary process. Dreams were not pure primary process, any more than psychotic processes are. What we actually see is a complex combination of diverse influences. Similarly Joyce's reactions and our interactions in the session I described earlier were not a pure expression of the zero process. Rather, as her defences gave way and as the traumatic memories emerged, we could catch a glimpse of some characteristics of the zero process more clearly than is usually the case. One of the key points of this paper is that the primary and zero processes are quite different ways in which the mind functions. Another key point is that we should not use our newer interest in and understanding of trauma to downplay Freud's momentous discoveries about the primary process, about the deeper emotional parts of the mind, about it's development related to childhood sexuality and aggression, and about it's dynamics. I feel that we will get a better understanding of trauma if we do not make everything trauma, but rather differentiate what is trauma from what is not. In fact, by comparing post-traumatic dynamics to the more classical drive/defense conflicts and issues that were first studied by Freud, I feel we can acquire a deeper understanding of both. So, in order to further this deeper understanding, I will first compare the zero process to the primary process with regard to a number of characteristics, and will then consider a few applications of the concept of the zero process.

\section{The Primary Process, the Zero Process, and the Secondary Process}

In the primary process there is an unceasing movement. Freud (1900) conceptualized that psychic energy in the primary process is freely mobile, always trying for a path of discharge, as compared to the secondary process, where the energy is bound. In the primary process energy and interest are easily displaced from one thing to another, and different contents can condense to form one image. Time does not effect the strength of wishes, or the nature of the contents of the primary process more generally, which led Freud to call the primary process timeless. There is no distinction made between fantasies and reality. We can observe a number of these processes at work in Cordelia's aggressive fantasies and death wishes towards her parents, which had been repressed at an early age, and emerged as part of our analysis of her trauma. She felt her anger had killed her mother, and on the other hand her mother's death increased her anger at her for abandoning her. But these rageful feelings were repressed. These wishes were generally projected onto other figures, such as a dog that landed on the ground and lunged at her in a dream, or the men who she was always afraid were breaking into her home. Here we see the displacement of the aggressive drive, as well as condensation of sexual and aggressive wishes in the invading men. Fantasies of murder scare her and make Cordelia feel as guilty as if she had actually done the deed, showing the lack of distinction between fantasy and reality. The wishes and fantasies were certainly unaffected by time, as fresh when they emerged in therapy as they were when she was a young child. The anger at her mother and father also showed up in the transference. The classic analytic transference is a product of a number of the characteristics of the primary process, showing the freely mobile energy which pushes for expression and discharge, and easily moves from one object to another, the lack of effect of time on the wishes, and the lack of differentiation between fantasy and reality.

The core of her zero process is also untouched by timefor instance Cordelia was always losing weight, always having stomach trouble. But the core of the zero process is timeless in a more frozen or mechanically repetitive manner. There is none of the ceaseless movement, also none of the displacement and condensation seen in the primary process. Rather there is a moment or a series of moments, frozen in time, endlessly running in a loop. As one of my other traumatized patients expressed it, "I just start to get upset and run through the reactions and feelings, once it starts. And then it's like it rewinds to the start like a videotape, ready to start up again. Its like I have no control over it once it starts, and no control knowing its going to start again sometime." The wishes and contents of the primary process are outside of time, and uninfluenced by it, or by the further experiences and learning that come with time. The memories of the zero process are uninfluenced by time, but they contain a piece of time frozen in a sequenced state, ready to unfold. This sequencing is absent from the primary process.

The primary process treats all psychic events as real, and in this sense there is a defective sense of reality. Since the zero process involves a past reality living out as if it were present, it does involve reality testing and a connection with reality, just one that is not judged correctly as part of the past. The primary process especially confuses fantasies and reality, while the zero process confuses past traumatic realities with present realities. It treats memories as present realities, while the primary process treats fantasied fulfillment of wishes as present 
realities.

While energies in the id, where the primary process reigns, are freely mobile and strive for discharge as they quickly jump from one thing to another, and those in the secondary process are more bound and measured in their movement, the zero process is a different thing altogether. On the one hand, there is certainly not easy movement and displacement - rather a fixed pattern of perception and affect and action and bodily changes is activated and lived out. We might say energies are rigidly bound, having only one form of dischargea fixed repetition. On the other hand, unlike the rational control in relation to means and goals imposed on the secondary process, the zero process has the restless striving for immediate discharge associated with the primary process. But the channels of discharge are tightly constrained. The id is powered by the drives of sexuality and aggression, which strive for discharge. The zero process is powered by not yet fully formed experience, which strives to finish forming and to become real.

Different authors have expressed various views about the source of the drive to repeat the trauma that is found in post traumatic states. The most commonly expressed psychoanalytic view is that the repetition is a way of turning a passively suffered trauma into something actively brought about, and this may be related also to a drive to master the trauma retrospectively. While there are efforts to master the trauma by this repetition, I do think there is evidence that the push to repeat the trauma is a drive in its own right, which originates with each traumatic experience. I have designated this the zero process drive, in analogy to the sexual and aggressive drives that originate in the emotional mind, in what Freud called the id. With the conceptualization of a zero process drive, we can also become aware of certain very characteristic zero process dynamics and conflicts, in which this drive to repeat is held in check and modified by various defences. Some of these defences are the regular ones described in other conflicts, such as repression and denial, while others use characteristics of the zero process. I have referred to these as zero process defences.

The best known zero process defence is dissociation, which uses the lack of integration of the various elements of traumatic memory, and of these memories with the person's regular memories, to keep the trauma separate from the person's regular experience. We can see the operation of dissociation very well in the cases presented previously, of Joyce and Cordelia, whose trauma lived in a separate world, at times intruding very forcefully. There are also a set of defences related to the characteristic of zero process memories of existing in some ways not as a past occurrence but as a future one. Because of the lack of construction of the traumatic experi- ence, the experience of the trauma not only lives as a present experience, but at the psychic level is actually always about to happen. Two defences that use this characteristic of the zero process are temporal shifting and zero process denial. In temporal shifting the traumatic memories are moved into the future, always about to happen, but never happening fully. For instance Cordelia was always afraid that someone who was ill would die, or that she would die, and was always trying to prevent it. Her traumatic loss of her mother lived in her future, always about to happen. This allows the trauma to be held at a distance, as with dissociation, but also gives the person hope, that it might all turn out differently. And Cordelia certainly had hope that she would be able to save these people, while of course she could not save her mother. Once one becomes aware of it, one can see that temporal shifting is common, in fact ubiquitous, in post traumatic states. Another similar defence, zero process denial, uses the not yet happened nature of the zero process to insist that the trauma is not real. Usually this operates at the feeling level. In Joyce's case, and this is quite universal in major trauma, we found that even after she had remembered and relived much of the trauma she still, in one part of her mind, did not take it as real. "I know we have gone over so many things that happened, and I believe that they did, but I still have this strange feeling of unreality and a feeling that it didn't happen and that I made it all up." I replied, "I wonder if you feeling that it hasn't happened is so strong because it's true. It hasn't happened yet. As we have seen in looking at them, the memories have not been fully put together and formed. They live in your fearful expectations for the future, and in your other reality and the other, wild haired you in the mirror. Our work is to make the reality finally real and finally happen fully by putting the pieces together." This example also demonstrates a specific technique of working with zero process defences, which is to not argue about their irrationality, but point out to the patient that what they think is actually true, but its just true of the other, traumatic, reality, with its strange ways of functioning.

A common and illuminating example of these differences that I have been discussing between the primary and the zero processes is borderline personality disorder (BPD). I am sure many of you have seen cases, often many cases, of this disorder. While people suffering from BPD can often function relatively well in neutral situations and do not have the breakdown in contact with reality and other basic mental functions of schizophrenics, in close relationships they demonstrate many of the characteristics of the primary process. One of the central dynamics in BPD is what has been called splitting. The person will see people as all good or all bad; they will either idealize and love someone, to the point of intense sexual excitement, or they will demonize and hate them. 
And this attitude can shift abruptly, so that the idealized person will all of a sudden become hated or denigrated. This is a not uncommon occurrence in therapy with such people, and this is why if a patient comes in and in the first session is sure you understand them perfectly and are so much better than those other terrible therapists they have seen previously, experienced therapists are not pleased, but rather know that they are in for a rough ride. At some point they will join the ranks of the terrible former therapists, and will be denigrated and hated as much as they were at first loved and adored. This borderline splitting involves a lack of integration between basic emotional trends, such as love and hate. We can compare this to post traumatic dissociation, for instance as seen in the cases of Joyce and Cordelia. This involves a lack of integration and active separation between bits and pieces of traumatic memories, and especially between the traumatic memories and the person's more regular memories and experience. Neither Cordelia nor Joyce demonstrated the splitting in their basic emotional life that is part of BPD, as shown by their ability to develop a stable and mature working relationship with their therapist. Thus borderline splitting and post traumatic dissociation are two very different things.

If one follows this line of thought, one can see a number of the characteristics of the primary process in BPD. These are different from characteristics of the zero process, seen in post traumatic states. (Of course people with BPD can also be traumatized and will then demonstrate zero process characteristics, but my point is that the defining features of BPD can be differentiated from the features of post traumatic disorder.) As another example, people with BPD will distort reality by confusing their wishes and inner anxieties with reality. They will say that people don't love them enough when really they have trouble maintaining stable love for others or themselves. Or they will say you were shouting at them when really you weren't, but they were unconsciously raging at you. In this the lack of differentiation between fantasy, emotional reality, and external reality of the primary process is in evidence. By comparison, when Cordelia would get panicky when someone was ill, as if they were about to die, or when Joyce was terrified of people coming into her room when her trauma had been triggered, they were demonstrating the confusion of a past traumatic reality, still alive as a dissociated experience, with present reality. This is the sort of reality distortion found in relation to the zero process.

Other characteristics of BPD could also be fruitfully studied as expressions of the dominance of the primary process. It is beyond the scope of this presentation to detail these. I have only waded into what is really too large a topic in order to provide a clinical illustration of the differences between the primary and zero processes and also to raise a theoretical point with important clinical implications. The prevailing view among most analysts with regard to BPD is that it is caused by developmental trauma, specifically failures in very early emotional care-taking. I do not share this view. I think there is good evidence both clinically and from heritability studies that the core deficits of BPD, especially the inability to maintain stable libidinal investment in the sufferer and in others, is an inherited one, with environmental factors playing an important part in whether the deficit manifest in more severe or less severe form. I think that the basic BPD deficit exists on a spectrum of severity. Again, it is beyond the scope of this lecture to discuss this controversial issue in depth, but I bring it up here because of its clinical consequences. BPD patients will tend to project their basic deficit, accusing first their parents and then others in their environment of not loving them enough. If one holds views on BPD causation related to deficits in care taking one may be tempted to analyze these accusations as markers of trauma, and to do trauma related therapy with such patients. This would be a clinical mistake. Not that sufferers from BPD may not have trauma in their histories, but to analyze their initial complaints as traumas would not address the basic structural deficit with regard to weak repressions and flooding of the ego by the id and the primary process. One would be reinforcing their projections rather than analyzing them and taking other measures to strengthen their weak egos. In treating BPD there is a powerful countertransference pull to take care of the poor suffering patient and to take their side against those they accuse, which can change over quickly into a hatred of the patient. In the case of post traumatic neuroses there is often a countertransference pull to avoid the trauma or at times even to pull back from full acknowledgement of how horrible the trauma was. I do not mean by this that therapists are not usually sympathetic and empathetic with their patients who have been traumatized. Rather, in analyzing their traumas, there is a countertransference tendency to avoid going too deeply into the details of the trauma, often with the rationalization that the patient will not be able to bear it.

Having outlined some of the basic features of the zero process by comparing it to the primary process, I would now like to apply this knowledge to other aspects of the psychoanalytic theory of the mind.

\section{Some Applications}

Many things can have a zero process version. As an example we could take mourning. Volkan (2014) describes perennial mourning in a number of his patients. For these patients the mourning is never over and the lost person is ever present. The mourner does not develop melancholia, but continues endlessly to engage with the object. Volkan describes a patient 
who talked to his dead his brother in his car driving to and from work, even though in other ways his reality testing was fine. He describes such patients dreaming endlessly about the person, but the dream always ends before the conflict is resolved. What is going on in such a perennial mourning? I would say it is a different way of getting stuck in mourning, as compared to depression. The comparison between depression and perennial mourning is instructive of the nature of the zero process, as compared to the primary process. In depression the mourning is repressed, because there are irresolvable conflicts, often over death wishes towards the person who has been lost. The mourning requires revisiting these wishes, but since they are under powerful repression, the mourning itself is dragged into the unconscious. At this point, in order to preserve the lost person, the whole process is internalized, the patient identifies with the lost object, and directs their hatred now at themselves, in the role of the object. As Freud (1917) says, "the shadow of the object fell upon the ego (p.249)." Because of the repression, the person no longer has access to certain memories and feelings about the lost person, and so the mourning, which needs this access, is brought to a halt, and the whole process comes to share in the timelessness and interminable nature of the primary process. In the cases of perennial mourning that I have seen, the loss was caught up in a traumatic process, and frozen in time. There is the endless perceptual repetition of the relation with the lost person. The mourner does not develop melancholia, but continues endlessly to engage with the object, for which Volkan says that the term introject would be appropriate. In perennial mourning, which could also be called zero process mourning, the accent falls on an ever present and ever repeated experience, which is what marks it out as a zero process phenomenon. It has a different kind of timelessness than depression-more repetitive of specific experiences, with the accent especially falling on the perceptual and affective aspects, while in depression drive and mood aspects, especially the continued aggressive attacks on the self, predominate.

As with perennial mourning, other aspects of mental functioning which have a strong experiential or reality aspect are worth investigating in terms of the zero process. Two types of symptoms which have traumatic fixation at their core are conversion symptoms and phobias. The clue with both of these is that specific real perceptual experiences are involved in the symptom. However, the proof is in the analysis of these symptoms. I presented some of this evidence in analyzing Cordelia's conversion symptoms of belly pain and of her right sided pain. Both were anchored by specific zero process memories. Other conflicts were contained in the symptoms, so that they were not just an expression of the trauma. Still, in all of the conversion symptoms that I have seen-and in my experi- ence these are much more common than is usually supposed -there has been a traumatic experience, frozen in an area of zero process functioning, relating to the physical expression of the symptom, even as other meanings and conflicts were then also expressed by it.

Immediately following a trauma, and in persisting posttraumatic stress disorder (PTSD), phobic avoidance of certain things connected the trauma are always present. In these cases the traumatic link is obvious, and leads generally to specific experiences at the height of trauma. In the case of other phobias, the traumatic reality is often hidden. Intuitively understanding the connection between phobias and trauma, people with a phobia will often look for a traumatic incident directly related to the phobia. For instance someone with a phobia of dental procedures will wonder if they had a bad experience at the dentist's. In these cases, the link is usually different. It has been understood by analysts since the early days that phobias use displacement, so that a drive conflict is displaced onto the phobic object. This is clearly a primary process mechanism, demonstrating one of the main feature of this mode of functioning. Freud (1909) described how five year old Little Hans displaced his fear of his father's retaliation for his oedipal and aggressive wishes onto horses, of which he became phobic. But in these types of phobias, in my opinion, there is always a traumatic core. Blum (2007) notes that in his history of little Hans Freud neglected a number of traumas, including a tonsillectomy operation, a very disturbed mother who beat Han's sister of only a year and a half, and also gave him enemas, and seeing a bloody miscarriage that his mother had at home. We find the reality of this zero process experience when we inquire more closely into what the person is really afraid of. For instance I have had a number of patients with phobias of going to the dentist but in each case, what really scared them was different. Whatever it was-for instance being held down and not able to move as they were damaged physically-the patient was not merely afraid that it would happen. Rather, they knew it would. It is this certainty of an event that marks something out as a phobia, and marks the phobia as a zero process symptom. Phobias are ways of containing and marking out the other reality contained in the zero process.

In using the term zero process symptom for phobias, and also for conversion symptoms, what are we asserting? Certainly not that everything about these symptoms relates to the zero process. But rather that there is a zero process core, a second reality, which acts as an anchor for other conflicts, and as an expression of them. I would propose a complemental series, as Freud (1916-1917) did with regard to hereditary vs. environmental factors, between the importance of the zero process and other factors and conflicts, in constructing symptoms. At one end, as can be seen with some phobias that im- 
mediately follow a trauma and are present in PTSD, the zero process memory predominates, and other conflicts play a minor part, while at the other end other issues may hold in place a zero process memory and use it for purposes of expressing themselves, but the symptom is largely caused by these other issues. The proof of what the various contributions are can only come in each case through the analysis of the symptom. As an example, in Cordelia's case it was necessary to move back and forth between the drive conflicts related to anger and oedipal sexual conflicts, and the actual traumatic occurrences and the aftermath of them, in order to get actual change in her conversion symptoms. There seemed to be a major contribution from both sides to the symptoms and reactions Cordelia had. We might think of Niederland's freezing patient having a symptom that had a predominant contribution from the zero process. This is not merely of theoretical concern. Taking various zero process and primary process dynamics into account is important clinically. Therapy can get stuck and start to go in circles if some factors, either traumatic fixations or drive conflicts, or factors such as developmental interferences, have not been taken into account and analyzed appropriately.

Just as there are zero process symptoms so also, there are zero process objects. Our understanding of internal objects may be clarified quite a bit by applying the idea of zero process internal objects. We would expect these to be linked to specific traumatic incidents, to have strong perceptual and action aspects, and to have a repetitive sort of interaction with others, or a set of reactions. Introjects generally have this character. The idea of the introject, as an internalization of an external object relation, that resides within the person and yet confronts them as something external, has not been well explained, in terms of its mechanism of formation and functioning. It can be contrasted with identification, in which our self representation and actions take on characteristics of those we love, or hate, and also with object representations, which are built up from regular memories and their abstraction and integration, belonging more to the primary and secondary processes. There is something of a continuum between more regular internal objects and zero process objects, depending on the contribution of each type of functioning. However, because of the drive like quality of zero process structures, which strive to become a full present experience, introjects seem to have, and in fact do have, a motivation independent of both the drives and of the regular ego interests of a person. Zero process objects are only part of the picture, certainly, but they may help to clarify it. Also, and importantly, we could inquire into the role of zero process objects within the superego as it develops. As with zero process symptoms, zero process objects are not just a pure expression of trauma. Rather, in the usual course of things, other representations of the object, oth- er issues and conflicts, become part of this introject. But what gives it a sense of reality as a present experience, and a sense of agency as something that acts apart from the person, are the zero process memories and zero process drives that are its core. These are what make it an introject.

There has been much interest in, and much written about, the intergenerational transmission of trauma. From a zero process point of view the key, and something that helps us understand the power of transmission, is that what is passed down are not memories or feelings or sets of beliefs, but a reality. The traumatized parent who draws the child into their world of past trauma has been described by many authors, especially in relation to the Holocaust, but also other traumas, individual and collective (Bergmann and Jucovy 1982; Brenner 2014; Volkan 2015). A number of concepts proposed in relation to intergenerational transmission convey the sense of a piece of reality being passed down and carried by the child. Volkan (2015) introduces the concept of a representation that is deposited in the child by the parent, while Kestenberg (1980) talks of transposition, in which the reality of the trauma that the adult suffered and the reality of the child's life are transposed. I think it is significant that these authors felt the need to come up with separate conceptualizations to point to something quite different than more regular mental actions. The concept of depositing suggests something quite concrete that is given over to the child, while Kestenberg (1980) specifically differentiated transposition from more regular primary/secondary process mechanisms such as identification. Treatment for such people needs to involve confrontation with reality-Grubrich-Simitis (2010) talks of the need for her patients, children of Holocaust survivors, to confront and test the reality of what happened to their patients. She notes that action in reality, and testing of reality, had very specific therapeutic effects: "The perception, recognition, and historicization of the Holocaust as external reality, together with the step-by-step process of symbolization in the context of the Auschwitz visits, proved to be essential preconditions for the progressive development of signal anxiety (p. 61)." These technical recommendations stem from the nature of the zero process as well, and show that its power passes down through the generations, an important finding also in considering socio historical issues.

From a therapeutic point of view, our insights into the nature of the zero process and symptoms, objects, and other processes, such as mourning, that have a zero process aspect, can guide us in our understanding of patients and in our interventions. In many situations such as these all sorts of conflictual, interpersonal, developmental, and transferential aspects will be addressed, but the more specific investigation of the zero process core, through analysis of avoidance, reluctance, re- 
pression, and zero process defences that keep it in place, often is not undertaken. Or not undertaken all the way to a true construction of the traumas. This can lead to a frustrating lack of progress in certain areas of the patient's problems. At the same time other sorts of conflicts and dynamics may be mistaken for trauma and only analyzed in relation to external impingements. Certainly helping in the development of a rational treatment for trauma, and a better understanding of how and when to use, and when not to use, trauma therapy techniques, is an important application of the concept of the zero process. In terms of the techniques themselves, properly comprehending the experiential nature of the zero process can make it more understandable why somatic techniques, as well as those using concrete perceptual tools, such as art, can be so powerful, and why talk by itself can often be so powerless, when dealing with the aftermath of trauma.

In this paper I have covered a lot of ground and introduced many new concepts and terms. I hope I have not overwhelmed you, certainly not traumatized you by giving you too much too fast. I have been developing these ideas as I write a larger book on trauma, and thought they may be of interest to a psychoanalytic audience. The basic idea, that trauma involves a shutting down of higher level ego functions, and that this in turn leads to a form of memory and functioning which exists in the present and future as immediate experience, rather than as regular memory, is relatively simple. This is what I have called the zero process. I hope I have stimulated your interest in this idea and also demonstrated that it has both theoretical and clinical usefulness.

\section{Acknowledgments}

This article was presented during 'Seminars with Dr. Fernando on trauma' with KAPA on 16-19 March, 2018.

\section{Conflicts of Interest}

The author has no financial conflicts of interest.

\section{REFERENCES}

Bergmann MS, Jucovy ME. Generations of the Holocaust. New York, NY: Basic Books;1982.

Blum HP. Little Hans: a centennial review and reconsideration. J Am Psychoanal Assoc 2007;55:749-765; discussion 851-852.

Brenner I. Dark matters: exploring the realm of psychic devastation. London: Karnac;2014.

Freud S. The interpretation of dreams. SE 4, 5. London: Hogarth Press; 1900.

Freud S. Analysis of a phobia in a five-year-old boy. SE 10. London: Hogarth Press;1909. p.1-150.

Freud S. Introductory lectures on psycho-analysis. SE 15, 16. London: Hogarth Press; 1916-1917.

Freud S. Mourning and melancholia. SE 14. London: Hogarth Press;1917. p.237-258.

Freud S. A note upon the 'mystic writing-pad'. SE 19. London: Hogarth Press;1925. p.225-232.

Grubrich-Simitis I. Reality testing in place of interpretation: a phase in psychoanalytic work with descendants of Holocaust survivors. Psychoanal Q 2010;79:37-69.

Kestenberg JS. Psychoanalyses of children of survivors from the holocaust: case presentations and assessment. J Am Psychoanal Assoc 1980;28: 775-804.

Volkan VD. Psychoanalysis, international relations, and diplomacy: a sourcebook on large-group psychology. London: Karnac;2014.

Volkan VD. A Nazi legacy: depositing, transgenerational transmission, dissociation, and remembering through action. London: Karnac;2015. 